\title{
An Unusual Presentation of an Intrapericardial Teratoma. A Case Report.
}

\author{
Arega Leta ${ }^{1}$, Anees Razzouk ${ }^{2}$, and Marx Genovez ${ }^{1}$ \\ ${ }^{1}$ Loma Linda University Health \\ ${ }^{2}$ Loma Linda University Medical Center
}

June 26, 2020

\begin{abstract}
We're presenting a unique case where a 2 month old female presented with URI symptoms at a local clinic and went into cardiac arrest after a nasopharyngeal swab test was done. Subsequent work up revealed she had an intrapericardial mass and a large pericardial effusion, which may have precipitated the arrest. The patient recovered after emergent pericardiocentesis and subsequent pericardial mass excision via sternotomy and pericardiectomy. The infant was recovering appropriately at her 4 month post-op follow up.
\end{abstract}

\section{Introduction:}

Intrapericardial teratomas come from three different germ layers ${ }^{3}$. They are very rare, (incidence of 0.05$0.15 \%)$ but very aggressive ${ }^{3,4,6}$. Their early detection and interventions are very important for effective management of the fetus. Most intrapericardial teratomas are attached to the aorta and tend to compress the right side of the heart structures ${ }^{1,5}$. As a result, fetuses can present with ascites, anasarca, and hydrops fetalis $^{2,5,8}$. Once evidence of hydrops is present in a fetus, the survival rate decreases when compared to a non-hydrops fetus (citation).

Therefore, early detection and good prenatal monitoring is essential. Prenatal period detection of intrapericardial teratomas is useful as interventions such as pericardiocentesis, or thoraco/pericardio-amniotic shunt can be done $2,5,8$. Tumor excision is the definitive treatment as its rapid growth could lead to right atrium, superior vena cava, and inferior vena cava compression ${ }^{7}$. This compression leads to ascites, hydrops fetalis, and/or anasarca. In our unique case a 2 month-old female did not present with the usual features of intrapericardial teratomas such as hydrops fetalis, anasarca, or ascites, and instead presented with cardiac arrest after an upper respiratory infection.

\section{Case:}

A 10 week old previously healthy female infant born via spontaneous vaginal delivery (SVD) at full term gestation, who was doing well until 2 months at which time she developed signs and symptoms of URI followed by cough but no fever. While in the medical office the patient went limp after a nasopharyngeal swab test and subsequent cardiopulmonary resuscitation (CPR) was done with return to spontaneous circulation (ROSC) after 2 minutes.

She was transferred to a local Emergency Department where she was intubated with a 3.5 uncuffed tube for concern of severe acidosis and work of breathing. A bedside ECHO showed a large circumferential pericardial effusion (Figure 1). The infant was transferred to Loma Linda University Children's Hospital (LLUCH) for higher level of care. 
At LLUCH a pericardiocentesis was done and a pigtail was sutured into position and placed on low intermittent suction. A CT Chest with contrast showed a $4.2 \times 3.6 \times 3.8 \mathrm{~cm}$ right sided pericardial/mediastinal hypodense mass with internal calcifications. The heart was shifted to the left due to mass effect. The mass was partially surrounding the aorta and SVC as well as the right pulmonary artery.

Echocardiogram showed normal biventricular systolic function with and EF of approximately $67 \%$ with no evidence of flow obstruction. A patent foramen ovale with a small shunt was also found. It also showed a multi-cystic mass appearing on top of the right atrium (Figure 2).

The mediastinal teratoma was excised via sternotomy and pericardiectomy

(Figure 3).

The tumor was found to be compressing the upper portion of the right atrium. The tumor was removed without the use of cardiopulmonary bypass. The teratoma seemed to have originated in the aorta because a $2 \mathrm{~cm}$ area of aortic adventitia needed to be shaved off (Figure 4).

Pathology report confirmed the mass to be a mature teratoma with tissue types from intestinal and respiratory epithelium, smooth muscle, skeletal muscle, cartilage, and glial tissue. There were no malignant or immature elements seen. The tissue from the aorta showed similar mature teratoma and the pericardium showed focal adhesions with no tumor infiltration (Figure $5 \& 6$ ).

The infant initially had problems feeding at her one and two month follow up visits, but was doing well at her 4 month post-operative follow up.

\section{Discussion:}

Our 10 week old female infant was a unique case as she was asymptomatic until she was 9 weeks old. It is unclear as to what specifically caused her to have cardiac arrest. We also suspect that when the baby limped and cried with sudden increase of intrathoracic pressure the cystic compoment of the mass might have ruptured to aggravate the pericardial effusion and precipitated the tamponade, resulting in cardiac arrest. It is equally possible that the large pericardial effusion found in bedside echocardiogram may have caused electric conduction disruptions in her heart leading to cardiac arrest. Review of the literature showed no case reports detailing an otherwise healthy infant presenting with cardiac arrest as a result of a cardiac mass. This case highlights the importance of close prenatal screening and to have a high suspicion for intrapericardial masses as a possible cause of cardiac arrest. Early identification allows for interventions such as pericardiocentesis, or thoraco/pericardio-amniotic shunt to be done in-utero allowing for prolongation of pregnancy ${ }^{2,5,8}$. On echocardiogram a patent foramen ovale with a small shunt was present, which may have prevented symptoms from presenting earlier.

\section{Conclusion:}

The case of our patient emphasizes the importance of routine meticulous prenatal ECHO screening. This may help identify pathologies such as intrapericardial masses early enough for an intervention. There is ample evidence that early identification of these conditions allow for effective interventions at the prenatal and post-natal period ${ }^{2,5,8}$. Our case highlights the importance for early identification as it is likely that our patient had evidence of a pericardial mass in-utero as most pericardial teratomas are present as early as 21 weeks gestation ${ }^{5,8}$. Furthermore, untreated or undiagnosed intrapericardial masses could present with sudden cardiac arrest as in our case, which could have a devastating outcome if not treated immediately.

\section{Notes on patient consent:}

Subject's parents were contacted via phone and asked to provide consent for usage of their child's case to be reported. Subject's parents provided consent for usage of the subject's case.

\section{Works Cited Page:}


1. Arvind, A. Successful management of a neonate with antenatally detected mature intrapericardial teratoma. (2019)

2. Banfiled, F. Intrapericardial Teratoma: A new and treatable cause of hydrops fetalis. (12/1980)

3. Heerema-McKenney A, Harrison MR, Bratton B, Farrell J, Zaloudek C. Congenital teratoma: a clinicopathologic study of 22 fetal and neonatal tumors. Am J Surg Pathol 2005;29:29-38.

4. Laquay, N. Intrapericardial teratoma in newborn babies $(12 / 2019)$

5. Rychik, J. Fetal intrapericardial teratoma: natural history and management including successful in utero surgery. $(12 / 2016)$

6. Solomon N. Adherent Intrapericardial Teratoma: A Case Report

7. Wall J, Albanese CT. Pediatric Surgery. In: Doherty GM. eds.CURRENT Diagnosis \& Treatment: Surgery, 14e New York, NY: McGraw-Hill; 2014. http://accessmedicine.mhmedical.com/content.aspx?bookid=1202\&sectionid=71529153. Accessed May 14, 2020.

8. Yuan, S. Fetal intrapericardial teratomas. (2/2019)

\section{FIGURES:}

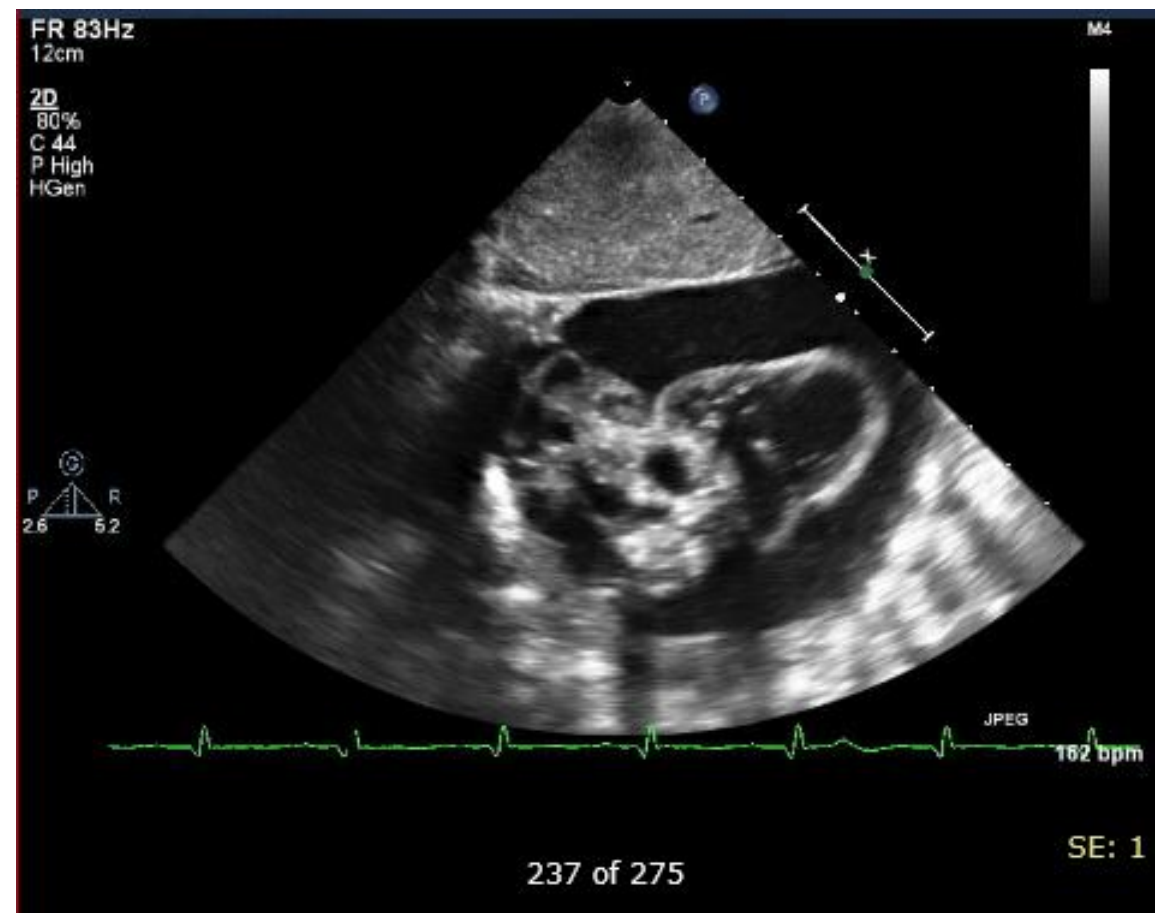

Figure 1. Large pericardial effusion evident in bedside ECHO. 


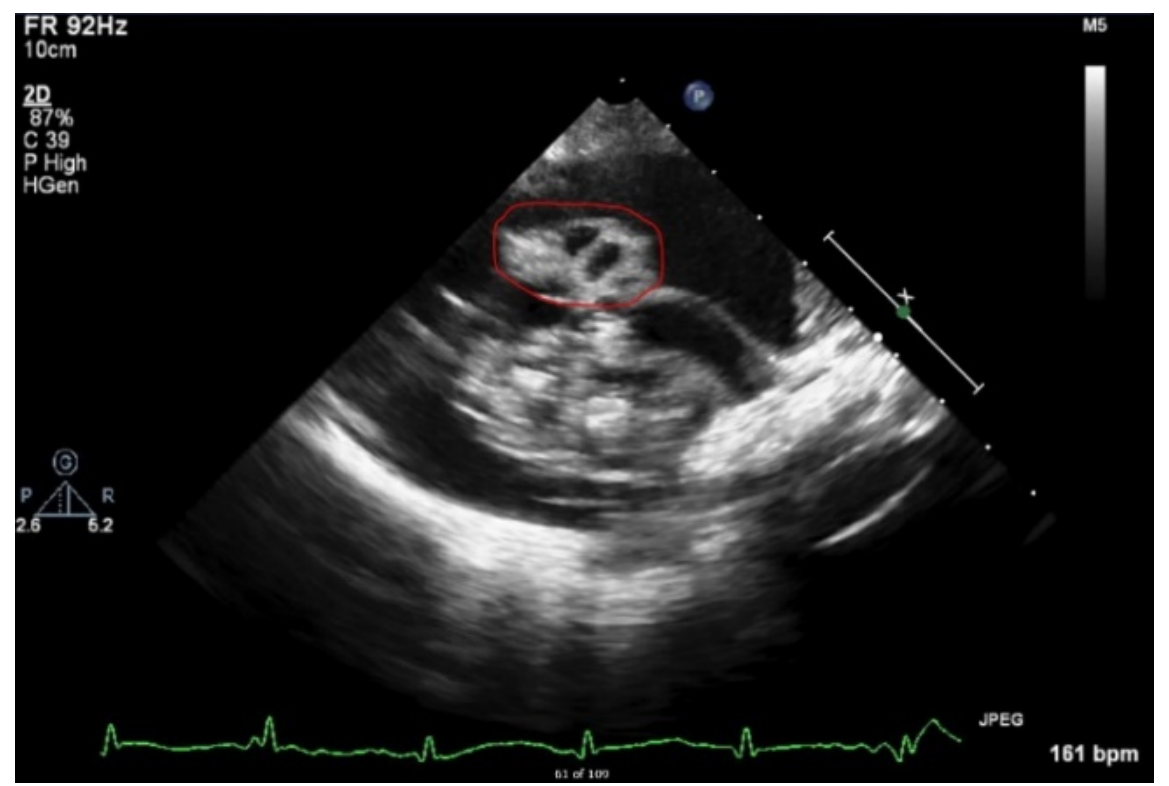

Figure 2. A multi-cystic mass was seen compressing the right atrium during ECHO evaluation.

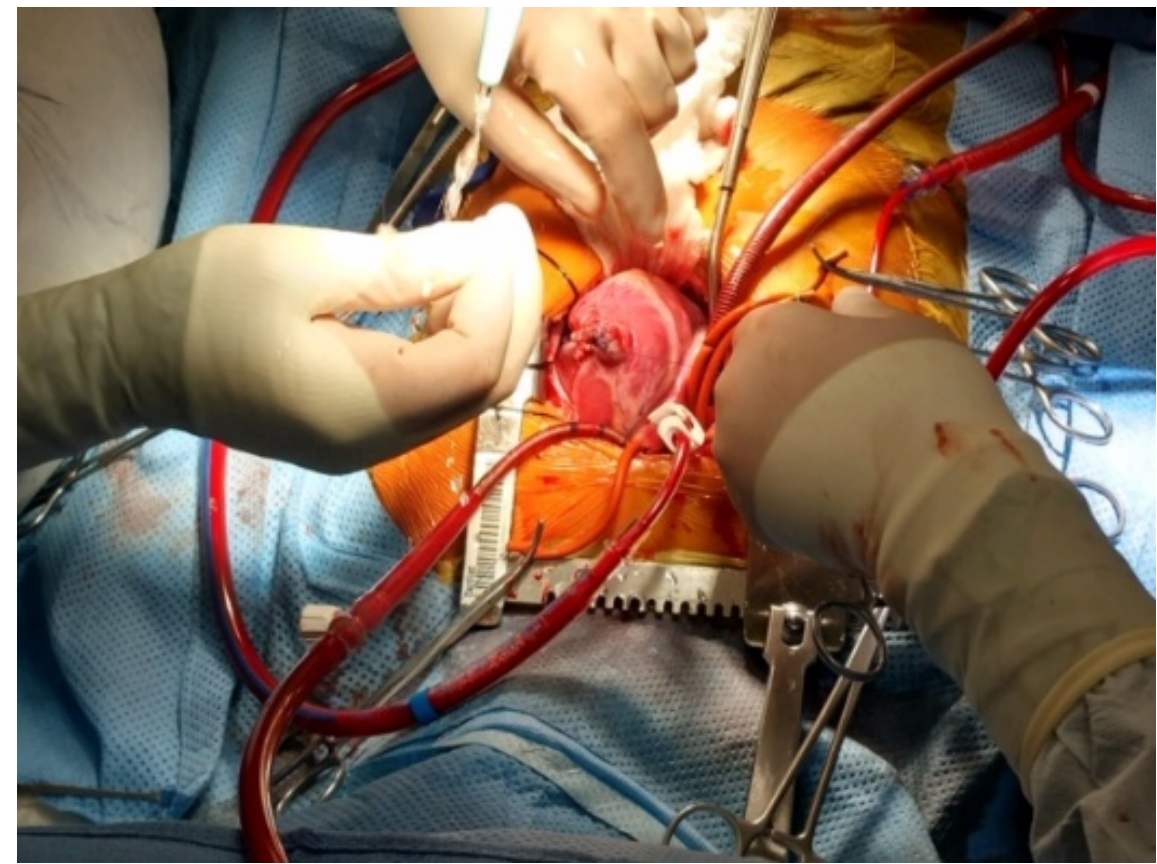

Figure 3. Closure of pericardium during anterior pericardiectomy with pericardial window to the left pleural space. 


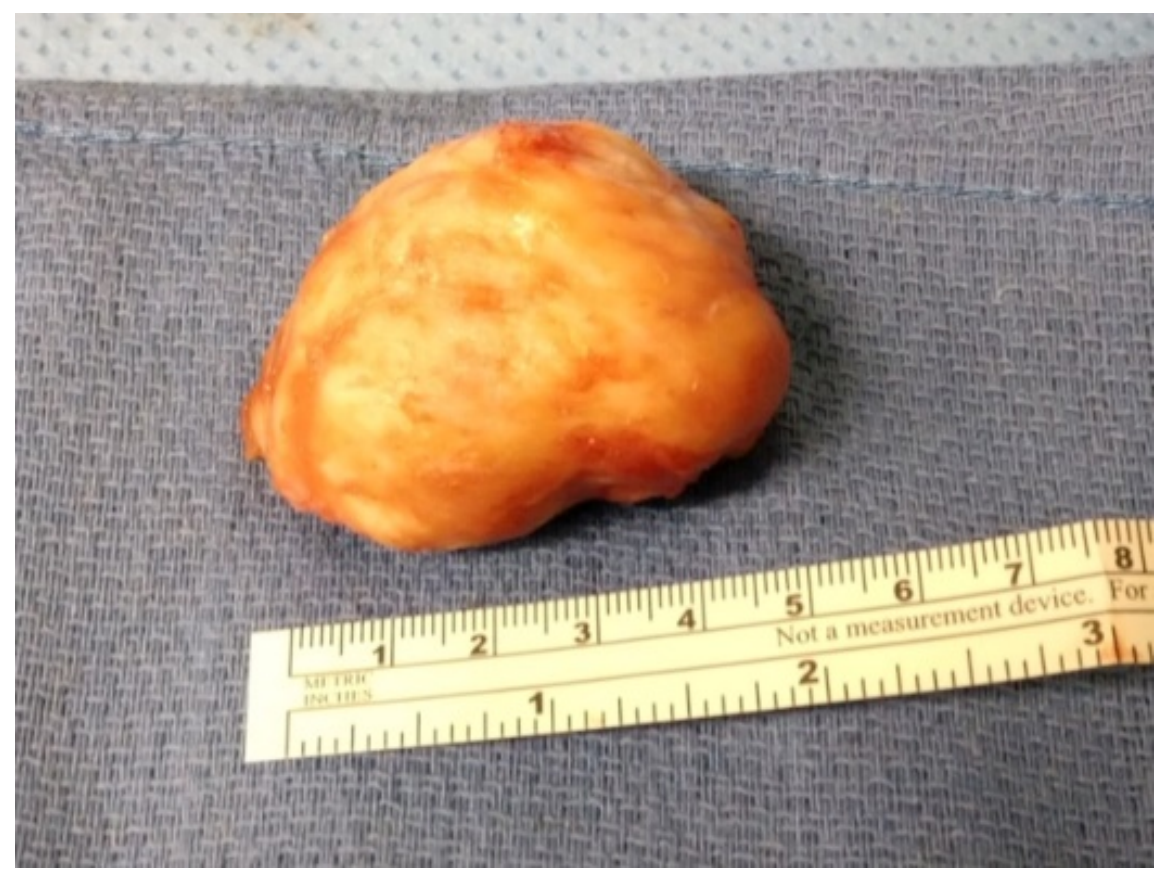

Figure 4. A $4.2 \times 3.6 \times 3.8 \times 5 \mathrm{~cm}$ multicystic, combined with soft and hard tissue mass adherent to the adventitia of the ascending aorta was excised at the level of the aortic root up to the distal ascending aorta along a $2 \mathrm{~cm}$ long segment.

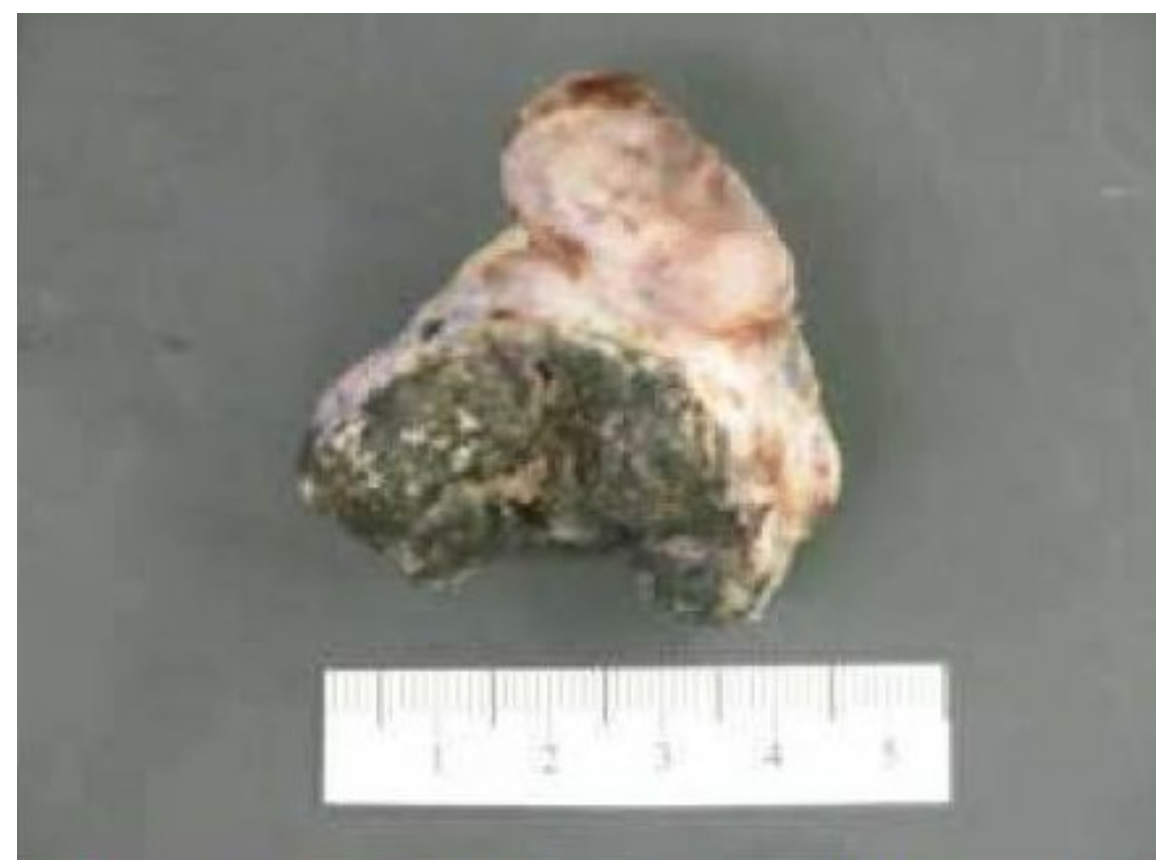

Figure 5. Pathology resection showing external surface of showing a mature teratoma with black ink on roughened margins. 


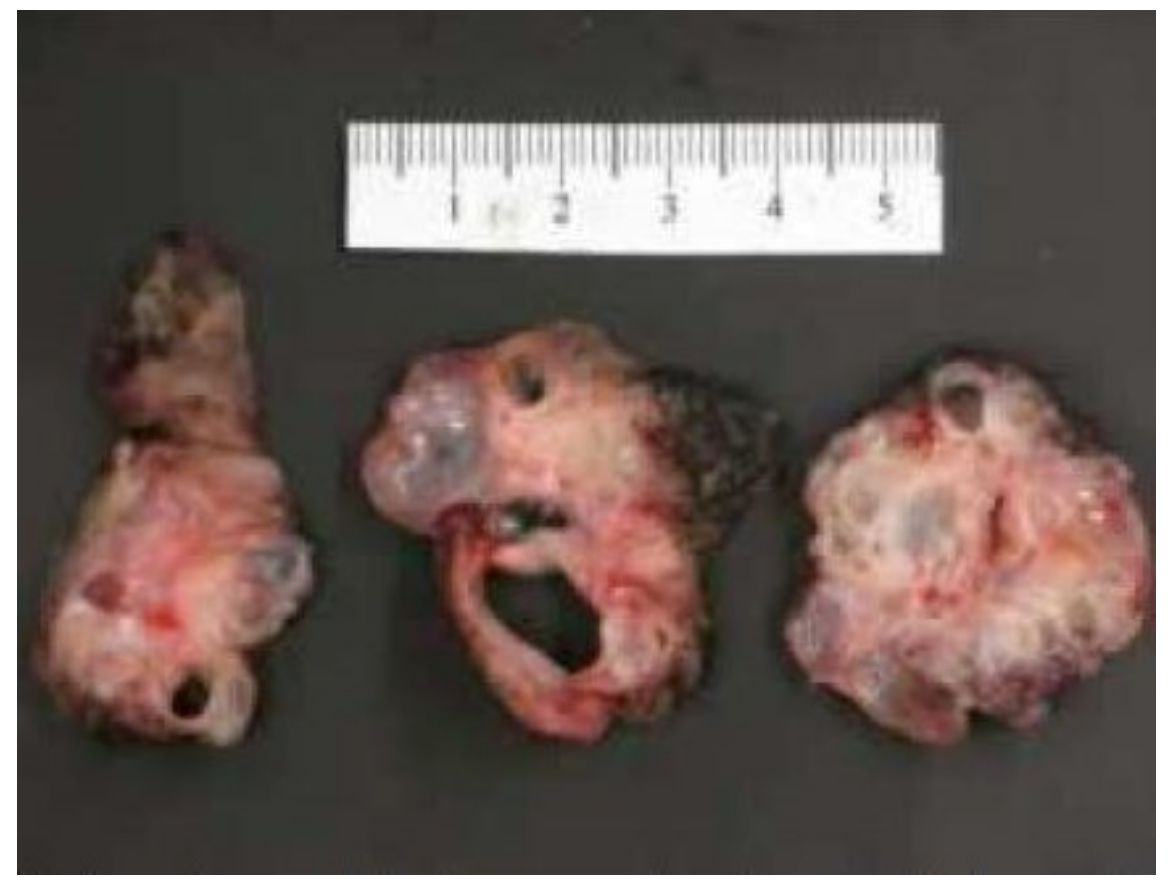

Figure 6. Pathology resection showing cut surface of the tumor, showing multiple cystic spaces. 